\title{
DISCRECIONALIDAD POLÍTICA EN LA ASIGNACIÓN DE RECURSOS: EVIDENCIA DE LOS GOBIERNOS REGIONALES Y LOCALES CHILENOS
}

\author{
Andrea Espinoza MuÑoz \\ Universidad Católica de Temuco \\ andreapaz.espinozam@gmail.com
}

Este estudio analiza la distribución de recursos llevada a cabo por los Gobiernos Regionales a diferentes municipios y organizaciones sociales en base al 2\% del Fondo Nacional de Desarrollo Regional sobre Cultura, Deporte y Seguridad. La investigación pretende contribuir a la literatura sobre políticas distributivas, gestión y asignación de recursos públicos, generando una discusión teórica respecto a la relevancia que adquieren los criterios empleados por el Gobierno Regional al transferir recursos. La hipótesis presume que la distribución del Fondo Nacional de Desarrollo Regional del 2\% responde a una lógica política más que a criterios de tipo socioeconómico y territorial, que benefician aquellos municipios y organizaciones sociales que se encuentran próximos al gobierno de turno. Para desarrollar esta investigación se tomaron los casos de cuatro regiones. Palabras clave: redistribución de recursos, Fondo Nacional de Desarrollo Regional, municipios.

\section{POLICY DISCRETION IN RESOURCE ASSIGNMENTS: EVIDENCE FROM REGIONAL AND LOCAL GOVERNMENTS IN CHILE}

This study analyzes the distribution of resources carried out by the Regional Governments to different municipalities and social organizations based on $2 \%$ of the National Fund of Regional Development on Culture, Sport and Security. The investigation tries to contribute to the literature on distributive policies, management and assignment of public resources, generating a theoretical discussion on the relevance acquired the criteria 
used by the regional government to transfer resources. The hypothesis presumes that the distribution of the National Fund of Regional Development of $2 \%$ answers to a political logic more than to criteria of socioeconomic and territorial type, that benefit those municipalities and social organizations that are near to the government of shift.. To develop this research the cases of four regions were taken.

Keywords: redistribution of resources, National Fund of Regional Development, municipalities.

\section{INTRODUCCIÓN}

La distribución de fondos públicos ha sido objeto de interés tanto para políticos como ciudadanos. A lo largo del tiempo su estudio ha presentado diversos enfoques, como teorías de corte económico, político y social. Por ejemplo, pioneras investigaciones de la década del setenta han destacado que el empleo de criterios políticos responde al estudio del comportamiento del gobierno en torno al gasto público. Poniendo en discusión las relaciones intergubernamentales (Oates, 1973), y las asignaciones como un proceso de maximización de votos esperados (Wright, 1974).

Dos modelos partidarios son los que configuran la asignación estratégica de recursos. Estos se basan en ganancias electorales por medio de la redistribución de beneficios hacia sus votantes. El primer modelo, de Dixit y Londregan (1996) y Lindbeck y Weibull (1993), predice que los beneficios entregados por los partidos políticos toman una dirección clara hacia la captación de los votantes indecisos. Agrega además, que la redistribución económica se produce en dos niveles distintos dentro del proceso político. El primero, de tipo programático, señala que la redistribución se apoya de programas redistributivos, es decir, en políticas sociales de largo alcance. Un segundo tipo, redistribución táctica, apunta a beneficios redistributivos, llámense estos subsidios o protecciones arancelarias, proyectos de construcción entre otras (Dixit y Londregan, 1996). La redistribución de beneficios se ve mediada por el modo en que son distribuidos estos recursos y a quienes se dirige. La competencia entonces, se apoya en lograr atraer el mayor nivel de electorado indeciso más que votantes leales. Asimismo, el modelo también se orienta a captar a los grupos económicamente más pobres, debido a 
que se presume una mayor susceptibilidad hacia los beneficios. El resultado de la densidad del punto crítico, la distancia entre partidos centrales en la elección municipal o de gobernante, y la renta, son las variables que determinarán la incidencia del modelo (Mediavilla, 2005: 181).

Por el contrario, un segundo modelo de redistribución partidaria, propuesto por Cox y McCubbins (1986:376), argumenta la distribución de los partidos políticos hacia tres tipos de votantes; estos son los grupos de apoyo, grupo de oposición, y el grupo de indecisos. Así, los partidos políticos compiten por el apoyo del mayor número de votantes a través de beneficios a particulares, y lo hacen apuntando a los grupos de apoyo, ya que estos son una inversión segura. Estos autores señalan que al orientar sus esfuerzos a los votantes leales produce un efecto clave dentro de la maximización de sus fines electorales. Puesto que estos votantes, para Cox y McCubbins (1986), se transforman en una pieza clave de retorno electoral, es decir, que aquellos grupos simpatizantes poseen mayor sensibilidad ideológica, por ende, es difícil que puedan desvirtuar sus convicciones, tal como los indecisos u oscilantes. Para comprobar estos supuestos el modelo se apoya de dos variables de carácter político; si el gobierno municipal es de igual partido al gobierno; y el porcentaje del electorado municipal que vota por el gobierno (Mediavilla, 2005: 176).

En este sentido, el presente trabajo se plantea analizar las dinámicas entre el gobierno regional y gobierno local. En función de poder discriminar cuán influyentes pueden ser los factores políticos dentro de los procesos de redistribución. Cabe preguntarse, ¿existe discrecionalidad política en los procesos de redistribución de los gobiernos regionales hacia los municipios? Alcaldes próximos al gobierno de turno y al intendente ¿presentan mayores niveles de transferencias respecto a municipios de la Concertación? ¿Otra clase de factores puede desplazar los intereses políticos? En base a aquello, presumimos que la distribución del Fondo Nacional de Desarrollo Regional del 2\% responde a una lógica política más que a criterios de tipo socioeconómico y territorial. Lo cual será respondido a través de un detallado análisis descriptivo como inferencial. 


\section{i. Redistribución TÁCtica}

Cuando la distribución de recursos emana del poder central o niveles de gobierno con alto poder decisorio, la lógica de redistribución de los recursos toma una mayor connotación política. Ya no son los partidos políticos los que mueven y direccionan recursos, sino el mismo gobierno, pero ¿con qué fin? ¿Serán criterios de eficiencia y equidad los que priman al pretender promover un mayor desarrollo a lo largo del territorio, o los factores políticos vuelven a posicionarse como en la práctica redistributiva de los partidos, con el fin de mantener y ampliar el electorado? Sin duda alguna, la redistribución táctica toma su mayor figura a este nivel. Si bien los modelos de Lindbeck y Weibull (1993), y Dixit y Londregan (1996); como el de Cox y McCubbins (1986), señalan que los beneficios van dirigidos a grupos previamente analizados, ya sean estos simpatizantes, opositores o indecisos, ambos modelos se enfocan en el mismo fin; maximizar su base electoral, ya sea para incrementar el nivel de apoyo al gobierno o disminuirlo, elevar su imagen ante la sociedad y buscar una alta identificación; o simplemente prolongar su injerencia social. Sin embargo, no nos explica del todo qué sucede a un nivel mayor. De tal manera que vamos a adentrarnos en esa descripción y en su tratamiento.

El Estado como encargado de velar por el cumplimiento de los requerimientos básicos, pretende dar cabida al mayor número de demandas posibles, a través de la implementación de políticas públicas, programas sociales, y por medio de cada uno de los servicios públicos destinados a garantizar su efecto. Lejos de detraer, genera un efecto rentable si pensamos en la consecuencia de políticas o programas de carácter popular de alto alcance, o beneficios a importantes segmentos con elevados niveles de impacto. En este sentido, cabe preguntarnos cuán sensibles son los sujetos a estos beneficios y cómo se traducen políticamente. En otras palabras, ¿se vale el gobierno de estos instrumentos para potenciar un mayor nivel de apoyo?

La evidencia muestra que la redistribución de recursos en este nivel ha sido significativamente puesta en práctica. Estudios como los de Dahlberg y Johansson (2002) contribuyen a fundamentar la existencia de motivos tácticos en la distribución de beneficios. En su investigación advierten una lógica de distribución del gobierno central a nivel local que coincide con criterios políticos. En sus hallazgos establece que los subsidios entregados, para fomentar los proyectos de 
desarrollo ecológico a municipios, se utilizan tácticamente por el gobierno de turno, estableciéndose que el gobierno invierte en aquellos municipios en los que existe una gran cantidad de votantes indecisos (Dahlberg y Johansson, 2002:24). Estos agregan al respecto que "observamos que esta variable política es claramente significativa en ambas estimaciones y tiene el signo positivo esperado: votantes más indecisos que hay en el punto de corte, mayor probabilidad de que un municipio reciba dinero del gobierno de turno" (Dahlberg y Johansson, 2002:17).

Se predice la aseveración respecto a la redistribución. No obstante, los autores también advierten que dentro de esta redistribución táctica toman importancia los factores sociales, que apuntan a las subvenciones en sí. Antaño, otra investigación de Johansson (1999) a los municipios suecos, frente a una reforma de corte social, reveló que la implicancia del factor político trasciende.

Otros resultados arrojan que la distribución de fondos públicos destinados a programas sociales también cae en la lógica de redistribución táctica para obtener beneficios políticos (Weitz-Shapiro, 2006). Su análisis sobre el programa Trabajar en Argentina determina que las variables políticas tienen una incidencia dentro de la distribución de fondos, principalmente la influencia del presidente y su entorno político, ya que caen dentro de la explotación de mecanismos políticos para conservar y mantener el respaldo de los sujetos (Weitz-Shapiro, 2006:143). Sumado a esta investigación, otro estudio al programa desarrollan Brusco, Nazareno y Stokes (2005), que anticipan que respecto a los recursos redistribuidos se manifiestan criterios netamente políticos usados tácticamente. Revelan los aspectos políticos que se emplearon en virtud de la evolución del programa; presentan que:

Los funcionarios argentinos a veces utilizan criterios discriminatorios en el reparto de bienes públicos, presumiblemente con el objetivo de movilizar el apoyo electoral. En el caso del Plan Trabajar, esta manipulación se intensificó notablemente cuando se acercaban elecciones. La estrategia de la manipulación fue la de buscar al votante marginal (indeciso o indiferente) antes que al fiel. (Brusco, Nazareno y Stokes, 2005:3).

La evidente intención política ha llevado a varios países latinoamericanos a incurrir en el empleo de la redistribución táctica. Perú es uno más que se suma a 
ello. La distribución de recursos económicos a través del programa FONCODES, creado para contribuir a la infraestructura social y mitigar la pobreza en proyectos basados en la comunidad, posicionó al gobierno de Fujimori dentro de los ejemplos de tácticas redistributivas. El uso del programa con fines políticos se produjo en puertas a las elecciones y a un referéndum celebrado años después de su mandato, donde se elevaron los recursos hacia los sectores populares a partir de 1993. En tanto que los gastos sociales se utilizaron como estrategia de captación a las provincias que habían disminuido su apoyo años anteriores (Schady, 2000:24). Conforme a estos resultados, Mediavilla (2005) en su estudio sobre transferencias discrecionales también constata la presencia de determinantes políticos en los procesos de reparto. Evidencia que las transferencias a diversos municipios argentinos se ve orientada por variables políticas, donde significativamente destaca el resultado de las elecciones y el alcalde del mismo partido del gobierno, vale decir, municipio que más se aproximaba al gobierno, mayor nivel de transferencias (Mediavilla, 2005:187).

Tal como se ha descrito, la evidencia pone de manifiesto que los criterios políticos son aplicados tácticamente en la redistribución de beneficios sociales. Es clara la existencia del uso táctico de diferentes instrumentos políticos, y su real contribución. Como también las consecuencias de dichos procesos, y quién los determina. No obstante, este empleo de la redistribución táctica podemos explicarla de acuerdo a un modelo alternativo. Grossman (1994) explica el reparto de transferencias por parte del gobernante. Adhiere que las subvenciones compran el apoyo de los votantes del estado y el capital político, como los recursos de políticos del estado y grupos de interés, que pueden ser invertidos en esfuerzos para aumentar el apoyo de los votantes para con el político federal (Grossman, 1994:296). A ello suma, que los políticos son maximizadores de votos interesados, es decir, que al atraer votos pretenden contribuir al gobierno central y a él mismo, tal como lo señala Mediavilla (2005: 177): "por ejemplo, que el alcalde, en caso de ser del mismo partido que el gobierno central, podría ejercer como un agente político que ayudaría a ganar las elecciones. Como resultado de esto se espera que reciban más recursos municipios con alcalde del mismo partido que el gobernante”. 


\section{Las transferencias gubernamentales en Chile: Fondo Nacional de Desarrollo Regional}

Este documento se centra en el estudio del Fondo Nacional de Desarrollo Regional (FNDR), precisamente en la distribución de un porcentaje de inversión del 6\%, que se subdivide en tres sectores: Cultura, Deporte y Seguridad; donde a cada uno de ellos se le determina un $2 \%$ del total del fondo para la realización de proyectos por parte de los municipios y organizaciones sociales que concursan. El FNDR es un Fondo de inversión que canaliza a las regiones del país parte de los recursos del presupuesto de la nación, permitiendo la materialización de programas y proyectos de inversión (SUBDERE s/f: 6). Creado durante el gobierno militar, pretende contribuir al desarrollo de infraestructura productiva como social de las regiones, con respecto a la Región Metropolitana. Una de las principales características de este fondo es que "las municipalidades preparan proyectos, y la distribución de los recursos a proyectos específicos corresponde al Gobierno Regional. El Consejo Regional resuelve la inversión de los recursos asignados en base a una priorización de proyectos propuesta por el Intendente" (Llancar, 2009:15).

El gobierno regional es quien debe representar los objetivos e intereses del gobierno nacional en la relación gestada con los gobiernos locales. Las municipalidades por otro lado, son quienes deben presentar sus demandas ante el gobierno regional para poder ser partícipes en la adjudicación de proyectos de inversión. En cuanto a la recepción de los proyectos, existen dos vías de postulación al FNDR, la vía sectorial y la vía del gobierno local.

En la vía sectorial los Servicios Regionales, desconcentrados de los ministerios, preparan proyectos; la respectiva Secretaría Ministerial realiza una primera selección de éstos y la deriva al Gobierno Regional, donde se seleccionan los proyectos, para ser presentados al Ministerio de Planificación (MIDEPLAN), el que realiza un análisis técnicoeconómico y recomienda los proyectos. Los proyectos municipales que postulen a financiamiento del FNDR, son revisados por la Secretaría Regional de Planificación y Coordinación (SERPLAC), para un posterior envío al Gobierno Regional, donde se seleccionan los proyectos para la realización del análisis técnico económico y recomendación por parte de MIDEPLAN (Llancar, 2009:16). 
De manera que nos encontramos frente a un proceso multinivel donde actores centrales, como regionales y locales interactúan en la configuración de este financiamiento. Para nuestro caso en particular, la distribución del 2\% del FNDR para Cultura, Deporte y Seguridad, si bien cuenta con claras instancias y reglas de participación, se transforma en un interesante foco de estudio, ya que se remite a un proceso de distribución más desconcentrado del nivel central. Los gobiernos regionales son ahora los actores clave dentro del proceso de asignación de los proyectos, ya que ellos más que nadie conocen mejor la realidad de su región y dinámicas de esta. En este sentido, la participación dentro de la redistribución de los recursos otorgados por el $2 \%$ puede transformarse, al igual que en los casos expuestos, en una evidencia más de redistribución táctica por parte del gobierno central, dado el carácter que adquiere la relación entre los gobiernos regionales y los municipios en la orientación de los fondos; más aún si estos poseen la naturaleza de concursables.

De acuerdo a la naturaleza concursable del fondo, tenemos que detenernos en las implicancias y en los actores dentro del proceso. Puesto que los proyectos por la vía municipal son recepcionados por el gobierno regional, aparece en esta instancia del proceso un actor fundamental. Los Consejeros Regionales (CORE), son quienes toman acto en el proceso de selección en conjunto con la figura del Intendente. Ambos seleccionan los proyectos presentados por los municipios como por las organizaciones sociales para una final asignación en conjunto con los organismos pertinentes. De modo que este hecho resulta clave al momento de pensar en un empleo de tácticas redistributivas por parte de los intendentes como consejeros regionales, simplemente porque ambos representan la visión del gobierno central, pero principalmente los intendentes. Estos deben reflectar la misión como los intereses políticos del presidente, y de acuerdo con ello, propiciar las condiciones para una futura continuidad, ya no para el presidente, sino más bien para su coalición. Por otro lado, los CORES por más que procuran un buen desempeño dentro de los aspectos administrativos, como legales, difícilmente pueden desprenderse de su aproximación política. Por ello, resulta vital estudiar la redistribución de los recursos que los gobiernos regionales asignan. Sin duda, esta noción se refuerza al pensar en el rédito político que generaría la financiación de proyectos cuando estos: 
Vienen a suplir la falta de inversión municipal, especialmente en municipios pobres, donde el FNDR se ha convertido en la principal fuente de financiamiento para ejecutar inversiones locales. Sin embargo, un alto porcentaje de proyectos es destinado a reparar, mejorar y reponer infraestructura en los sectores de salud y educación, de manera que el FNDR representa un subsidio tanto a la inversión municipal como a la falta de recursos de los municipios para mantener y conservar adecuadamente la infraestructura de los servicios traspasados (Llancar, 2009:17).

Ciertamente la finalidad del FNDR viene a compensar la serie de falencias estructurales como sociales dentro del contexto regional, por lo que analizar la existencia de criterios políticos se convierte en un fenómeno interesante de estudio.

\section{LA INVERSIÓN DEL 2\% Y SU DISTRIBUCIÓN REGIONAL}

Para llevar a cabo el estudio elegimos cuatro regiones, estas son: Región de Atacama (III), Región Del Libertador Bernardo O’Higgins (VI), Región de La Araucanía (IX) y, Región de Los Lagos (X). La selección fue de acuerdo a los montos y distribución de estos en cada región; siendo la región de Atacama la con menor nivel de inversión. O’Higgins y La Araucanía por concentrar mayores promedios de distribución, y un nivel promedio de inversión. Y Los Lagos por ser la región con el nivel de inversión superior a las demás regiones. Se utilizaron las cifras del año 2012, esto permitió tener 1.415 observaciones (suma de todas las iniciativas emanadas por los gobiernos regionales). El promedio de monto para cada proyecto respecto a su región, se presenta en el cuadro 1.

\begin{tabular}{c|c|c|c}
\hline CUADRO 1. & Monto efectivo del 2\% para cada región & \\
& Proyectos & Promedio & Total \\
\hline Atacama & 302 & 3.527 .868 & 1.065 .416 .086 \\
\hline O’Higgins & 210 & 8.311 .944 & 1.745 .508 .332 \\
\hline Araucanía & 195 & 8.002 .443 & 1.560 .476 .396 \\
\hline Los Lagos & 708 & 3.277 .919 & 2.249 .966 .816 \\
\hline
\end{tabular}

Fuente: Elaboración propia con datos de los respectivos gobiernos regionales. 
Podemos observar que la Región de Atacama, si bien tiene un mayor nivel de iniciativas que las regiones de O'Higgins y La Araucanía, posee el menor monto total destinado a estos proyectos. En contraste con la Región de Los Lagos que posee el nivel más alto en financiamiento para estas iniciativas. Esto se explica simplemente por la magnitud de cada región y su situación en particular. Por tanto, cada región posee un nivel de recursos independientes entre sí. De este modo, los promedios regionales para la asignación de un proyecto varían de una región a otra. La Araucanía y O’Higgins distribuyen montos más altos, llegando a los 8 millones por proyecto. En cambio, las regiones de Atacama y Los Lagos poseen un promedio cercano a los 4 millones. Sin embargo, la distribución de estos proyectos responde a una descripción de mayor nivel.

De los 1.415 proyectos adjudicados por las diferentes comunas el 19,15 por ciento corresponde a proyectos de seguridad. El 34,56 por ciento a proyectos de cultura y el 46,29 por ciento, casi la mitad de ellos, conciernen a proyectos de deporte. No obstante, estas cifras varían al observar la asignación en cada sector, ya que las iniciativas que reciben un promedio de monto mayor son las de seguridad con 6.875.176 millones, seguidas por cultura con 5.122.924 millones y, por último deporte con un promedio de 3.439 .824 millones por proyecto. En los casos regionales la tendencia resulta invertida, a excepción de la Región de Los Lagos (X). El promedio de monto por proyecto en torno a cada sector enseńa que las tres regiones restantes (III, VI y IX) tienen un promedio de monto mayor en cultura que en seguridad.

Sabemos que los proyectos van ligados a una comuna determinada, pero ¿quiénes se adjudican estos? Como señalamos en un comienzo, los fondos son concursables y puede participar cualquier ente público como privado. En efecto, las municipalidades son los organismos públicos que participan en esta asignación, las diferentes organizaciones sociales por otra parte, comprenden a los diferentes cuerpos civiles organizados que pretenden adjudicarse algún proyecto. Para determinar qué organizaciones son las que reciben un mayor reparto, creamos la variable tipo de organización, la que agrupa los diferentes tipos de organizaciones; estas son: municipio, agrupación social, clubes, junta de vecinos, fundación, y otro. Los datos de acuerdo a las medias presentan que los mayores flujos pasan hacia los municipios, seguidos por las fundaciones y, en tercer lugar las juntas de vecinos. Estas tres organizaciones tienen mayores promedios de montos. 
Esto se explica para el caso del municipio, por ser el ente público encargado de formular las iniciativas de inversión para la comuna, de modo que es el organismo que adquiere más relevancia en comparación con las demás organizaciones. Constatamos esto de igual forma para todas las regiones, y los datos señalaron que los municipios, como ya hemos indicado, se llevan el principal monto dentro de la repartición. En contraste con ello, las organizaciones sociales con menor monto promedio son clubes y las agrupaciones sociales.

Hasta el momento hemos descrito la distribución de los recursos de acuerdo a una lógica de tipo organizacional: qué organizaciones reciben más fondos, a qué tipos de proyectos responden estas organizaciones, etc. Por lo cual, pensar de qué otra manera puede responder la distribución nos lleva a plantear la importancia de otras variables.

En cuanto a la distribución urbano-rural. Los datos muestran que a mayores niveles de ruralidad no hay relación en la asignación de fondos, es decir, que en comunas con alto nivel de ruralidad donde podría esperarse mayor reparto no se produce una distribución, al contrario. Las comunas con mayor ruralidad menos recursos reciben. Mientras que al analizar el nivel de pobreza se obtiene que comunas más pobres superan en asignación a comunas con mayor nivel de recursos. Este promedio de pobreza se aplicó a todas las regiones, tomando como corte el 14,4 por ciento de nivel de pobreza nacional según la encuesta CASEN 2011. Los montos están relacionados con los niveles de pobreza. Así, para las comunas que se encuentran bajo el nivel promedio de pobreza nacional, menor promedio de asignaciones en comparación con las comunas más pobres (Gráfico 1).

Gráfica 1: Promedio del monto de acuerdo al nivel de pobreza 2012

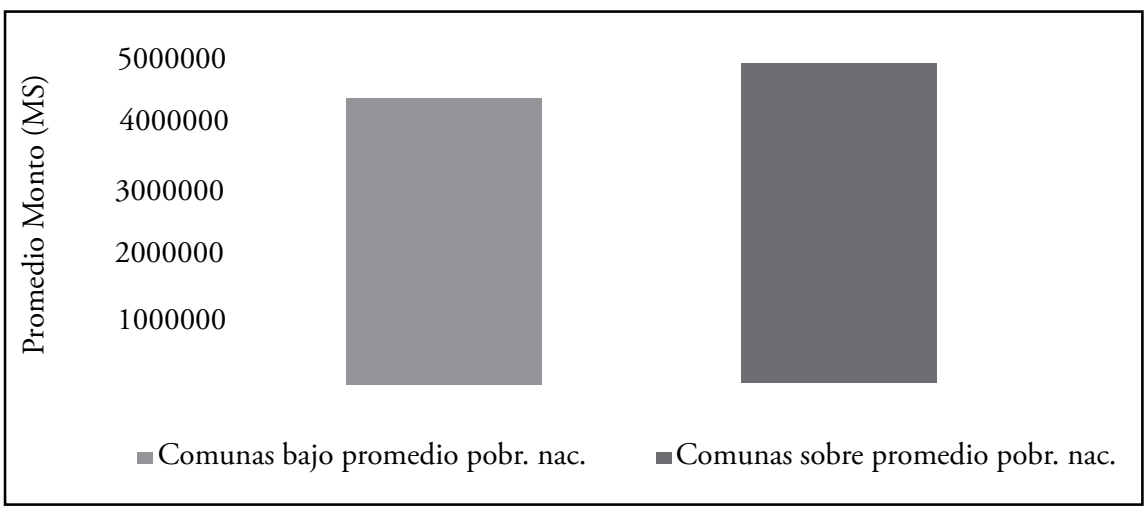

Fuente: Elaboración propia con datos de los respectivos gobiernos regionales. 
En el gráfico 2 se observa la distribución de los montos totales de inversión de acuerdo al porcentaje de pobreza de cada comuna. El mayor número de comunas se concentra en un nivel de pobreza del 10 al 20 por ciento con un promedio de monto asignado entre los 10 millones. Sin embargo, existen casos en que la asignación pasa de los 20 millones dentro de ese parámetro. La menor cantidad de comunas, ubicadas en los mayores porcentajes de pobreza (del 27 al 37\% aproximadamente) reciben dos sumas de montos, bajo los 10 millones para la mitad de los casos, y sobre los 10 millones para el resto.

\section{Gráfico 2: Distribución del monto y pobreza}

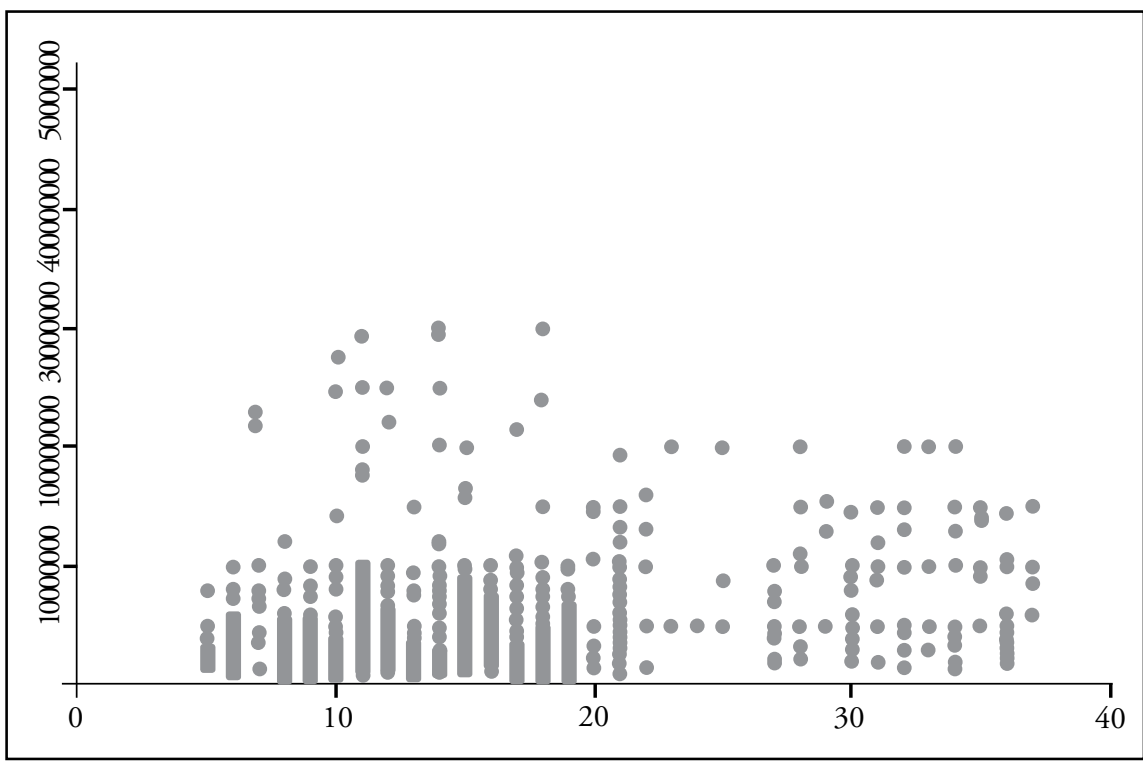

Fuente: Elaboración propia con datos de los respectivos bobiernos regionales y SINIMI.

Teniendo presente este hecho nos planteamos dos cuestiones relevantes. Primero, existe una voluntad en torno a la distribución de los recursos hacia las comunas más pobres en contraste con las de mejores ingresos. A ello respondemos, advirtiendo que existe una discrecionalidad socioeconómica dentro de la repartición de los fondos. Segundo, de acuerdo a la distribución del fondo y su proceso de aprobación, los factores políticos pueden diferenciar los resultados. En este sentido, 
las variables partido político del intendente, partido del alcalde, números de consejeros regionales próximos a la alianza, número de consejeros regionales próximos a la concertación, y el monto; exhiben diversas relaciones y resultados. Pese a ello, no todos tienen la misma significancia. De manera que, el partido del alcalde resulta obtener las evidencias más interesantes.

\section{Gráfico 3: Monto vs Alcaldes}

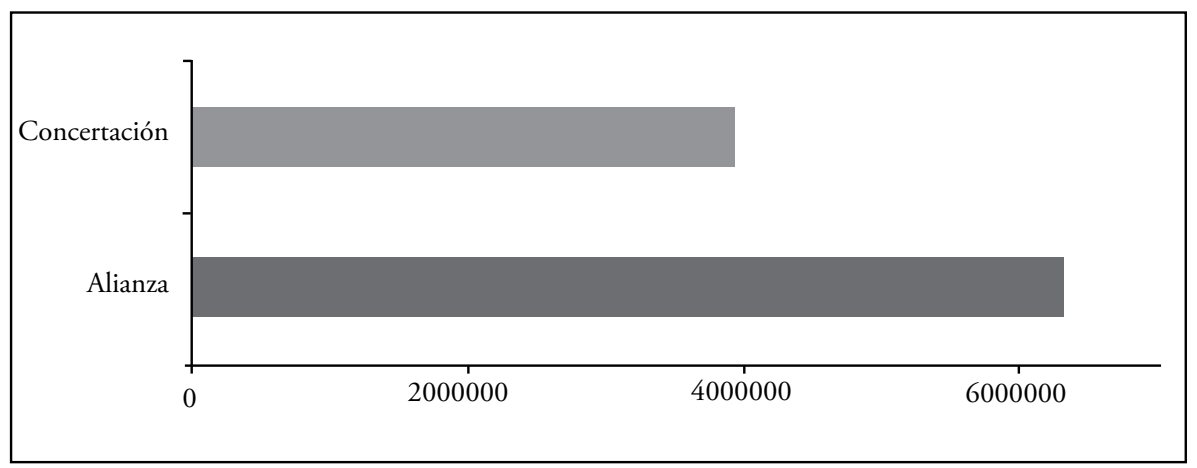

Fuente: Elaboración propia con datos de los respectivos gobiernos regionales y SLRVLL.

Las asignaciones en comunas donde un alcalde de la Concertación preside el municipio en comparación con un alcalde de la alianza resultan ser menores. Las medias del monto exhiben que los municipios de la alianza reciben una mayor suma en comparación con los de la concertación. Los datos mostraron que para los alcaldes de la Concertación, el promedio de la asignación casi bordea los 4 millones de pesos, mientras que los municipios de la alianza pasan de los 6 millones. Este hecho vuelve a ocurrir si comparamos las medias de los repartos entre ambos alcaldes en función del logaritmo del monto. 1 millón cuatrocientos para los alcaldes de la concertación, y 1 millón quinientos para los de la alianza. Si bien la distribución es más equitativa, aún deja a los municipios de la concertación bajo los alcaldes de la alianza.

Es clara la existencia de discrecionalidad política en la redistribución al pensar que el gobierno de turno es próximo a todas las comunas donde el municipio es encabezado por un alcalde de la alianza. Más aún, si el reparto, como muestran los datos, es más cercano a los alcaldes de la Alianza sobre los alcaldes 
de la Concertación. Esta noción se refuerza al indicar que a mayor número de consejeros regionales de la concertación, menores fondos le son distribuidos en comparación a mayor número de consejeros de la Alianza. Sobre estos términos descubrimos dos situaciones. La primera, advierte que la organización que más se beneficia (después de los municipios) en comunas donde el alcalde es de la Concertación son los clubes con un 23,41 por ciento, y la que menos aporte obtiene es la fundación con un 2,9 por ciento. Para el caso de las comunas con alcalde de la Alianza los resultados se reproducen en un 22,51 por ciento y 5,6 por ciento respectivamente.

Por otro lado, los resultados según el tipo de proyecto, demuestran que para las comunas con alcalde de la Concertación el sector más importante y que mayor aporte recibe, radica en cultura con un 40,9 por ciento, le siguen de cerca los proyectos en deportes con el 35 por ciento; en tanto, el sector que menos reparto obtiene es seguridad con un 24,1 por ciento. Por el contrario, las comunas encabezadas por alcaldes de la Alianza presentan una mayor inversión de iniciativas en seguridad, que alcanza el 38,92 por ciento. Deporte se queda en el segundo lugar con un 32,14 por ciento, mientras que cultura solo absorbe un 28,94 por ciento de las distribuciones.

\section{Modelo y demostración}

Para continuar con la descripción presentamos el primer modelo construido. La variable dependiente la constituye el monto (Log. del monto) asignado a cada comuna por proyecto. Las variables independientes corresponden al nivel de pobreza, nivel de ruralidad (ambas variables dummy que toman valor 1 los casos con alto nivel, y valor 0 para bajos niveles), alcalde de la concertación, variable dummy que indica alcalde de la Concertación (adopta valor 1) versus todos los demás (adopta valor 0), otra variable dummy que discrimina entre consejeros de la Concertación (adopta valor 1) y todos los demás (adopta valor 0 ), lo mismo para las variables proyecto en seguridad, proyecto en cultura, fundación y municipio (todas dummy).

Ya hemos observado los principales resultados descriptivamente y vemos que se desarrollan diferentes tipos de discrecionalidad. Al respecto, vamos a corroborar 
inferencialmente estos hallazgos haciendo una selección de las variables que mejor se aplican a estos hechos. Para ello, vamos a presentar a través de una regresión multinivel la implicancia de cada una de las variables. Los resultados se exponen en el cuadro $\mathrm{N}^{\circ} 2$. Aquel arrojó que la variable pobreza presenta signo positivo y significativo, lo que nos señala su fuerte relación con la distribución de recursos.

\section{Cuadro $\mathrm{N}^{\circ}$ 2. Primer Modelo regresión multinivel}

\begin{tabular}{|c|c|}
\hline Variable dependiente: Monto & \\
\hline Comunas bajo promedio pobreza nacional & $\begin{array}{l}0.265^{* * *} \\
(0.0688)\end{array}$ \\
\hline Comunas bajo promedio ruralidad & $\begin{array}{l}-0.197^{* *} \\
(0.0989) \\
\end{array}$ \\
\hline Alcalde Concertación & $\begin{array}{l}-0.171^{* *} \\
(0.0684)\end{array}$ \\
\hline Consejo Concertación & $\begin{array}{c}-0.192^{* * *} \\
(0.0242)\end{array}$ \\
\hline Proyecto seguridad & $\begin{array}{l}0.412^{* * *} \\
(0.0354)\end{array}$ \\
\hline Proyecto cultura & $\begin{array}{l}0.329^{* * *} \\
(0.0286)\end{array}$ \\
\hline Fundación & $\begin{array}{l}0.275^{* * *} \\
(0.0885)\end{array}$ \\
\hline Municipio & $\begin{array}{l}0.500^{* * *} \\
(0.0306)\end{array}$ \\
\hline Constante & $\begin{array}{c}16.74^{* * *} \\
(0.242)\end{array}$ \\
\hline $\begin{array}{l}\text { Número de observaciones } \\
\text { Número de grupos }\end{array}$ & $\begin{array}{c}1.415 \\
102\end{array}$ \\
\hline
\end{tabular}

Fuente: Elaboración propia con datos de los respectivos gobiernos regionales y SERVEL. ${ }^{* * *}$ significativo al $0.01,{ }^{* *}$ significativo al 0.05 .

Eso se encuentra directamente relacionado con potenciar el desarrollo en zonas con mayor vulnerabilidad, de ello que comunas con niveles más altos de pobreza reciban mayor cantidad de recursos en comparación con comunas de mejor ingreso. Como explica la teoría estos factores socioeconómicos son determinantes en los procesos de redistribución. Pese a ello, nos encontramos contra lo esperado, 
ya que la pobreza se presenta determinante al pensar en el proceso de asignación. En tanto, la variable ruralidad se presenta significativa pero negativa. Ello explica que comunas con mayor porcentaje de ruralidad reciban menos sumas. Esto significa que las capitales regionales y las zonas urbanas son las que absorben los mayores niveles de inversión.

En cuanto la variable alcalde de la Concertación, si bien es significativa y de signo negativo, resulta ser poco determinante en las asignaciones. Alcaldes de la Concertación reciben menor cantidad de fondos que alcaldes de la Alianza. Si recordamos a Grossman (1994), este advierte que las distribuciones son determinadas por la aproximación política. De manera que los alcaldes de la concertación reciben menor cantidad de recursos al representar el sector opositor al gobierno de turno. Sumado a que el partido del intendente (RN) predomina en 3 de las cuatro regiones. Este hecho resulta ser relevante, ya que en comunas con alcalde del Partido Radical Social Demócrata (PRSD) e intendente de Renovación Nacional (RN), tienen un promedio de monto menor que comunas con alcalde de la Unión Demócrata Independiente (X Región).A estos resultados, se suma la variable consejeros Concertación, esta reafirma la convicción sobre la proximidad política, ya que estos perciben menores sumas de inversión en contraste con los consejeros de la Alianza. Sobre esto Mediavilla (2005) coincide, argumentando que la trascendencia de la proximidad política es clave al estar en un contexto donde el gobierno central y los gobiernos locales estén en sintonía (Mediavilla, 2005:187). Ello explica la diferencia entre los fondos para ambos consejeros, resultando los de la Alianza con mayor ventaja.

Por otro lado, constatamos que la variable proyecto en seguridad es significativa y positiva. Esta se explica por las importantes sumas de inversión. Como se describió anteriormente, los promedios en proyectos de seguridad son superiores a los demás sectores. Pero una cosa interesante de aquello, es la relación de los proyectos de seguridad con alcaldes de la Alianza. Esta relación puede interpretar los mayores promedios de inversión en estos proyectos, ya que como se demostró los alcaldes de la Concertación reciben menos recursos que los de la Alianza, por ende podemos inferir una cierta consecuencia de aquel vínculo. Empero, la variable proyecto en cultura también se transforma significativa y positiva para el modelo, pero en comparación con seguridad es en menor medida. Aun así, es una variable que determina niveles considerables de transferencias. Esto se debe a que 
casi la mitad de estos proyectos corresponden a la Región del Libertador Bernardo O’Higgins y la Región de Los Lagos, además estas dos regiones son las que mayor nivel de inversión destinan al financiamiento de los proyectos. De esta forma los proyectos en cultura adquieren una posición definida en la distribución.

En cuanto al tipo de organización, se aprecia que la variable fundación se aplica para comprender el modelo. Esta demostró ser significativa en la distribución, ya que obtiene el primer lugar en torno al promedio de monto asignado entre los tipos de organizaciones. Este resultado va de acuerdo al nivel de envergadura y monto de los proyectos, siendo estos los que obtienen cifras considerables de asignación en comparación al resto de las organizaciones (a excepción de los municipios). Por último, la variable municipio demostró ser la más significativa dentro de la categoría de tipos de organización. Estos, al representar el gobierno local, mueven las mayores cifras en todos los tipos de proyectos. De tal modo que resultan ser claves dentro de la distribución, ya que los municipios con alcaldes de la Concertación manejan menos niveles de monto, en contraste con los municipios de la Alianza que los superan reñidamente.

El segundo modelo, presentado en el cuadro $\mathrm{N}^{\circ} 3$, mantiene todas las variables menos alcalde Concertación, ya que es reemplazada por alcalde Alianza. Al igual que en el primer modelo la variable pobreza se vuelve relevante dentro de la distribución, pero en este modelo es más significativa que el anterior. Esto se explica por el alto nivel de susceptibilidad hacia los beneficios que se presume poseen los sectores con mayores niveles de pobreza (Dixit \& Londregan, y Lindbeck $\&$ Weibull). En cuanto a la ruralidad, se mantiene la significancia negativa que representa las menores cantidades de fondos que reciben las comunas más rurales. Para los consejeros de la concertación sucede lo mismo. Reciben menores sumas de monto, ya que tienen un impacto negativo dentro del proceso de distribución. $\mathrm{Al}$ contrario, los proyectos de seguridad y cultura absorben mayores montos y reproducen los resultados del primer modelo. Ambas variables son positivas y significativas. No ocurre lo mismo para la variable fundación en este modelo. $\mathrm{Si}$ bien en el modelo anterior resulta ser significativa ahora no. En tanto, el municipio reproduce el resultado y muestra ser muy significativo dentro de las asignaciones. 


\section{Cuadro $\mathrm{N}^{\circ}$ 3. Segundo modelo regresión multinivel}

\begin{tabular}{|c|c|}
\hline Variable dependiente: Monto & \\
\hline Comunas bajo promedio pobreza nacional & $\begin{array}{l}0.315^{* * *} \\
(0.0942)\end{array}$ \\
\hline Comunas bajo promedio ruralidad & $\begin{array}{c}-0.268^{* *} \\
(0.112) \\
\end{array}$ \\
\hline Alcalde Alianza & $\begin{array}{r}-0.0864 \\
(0.0956)\end{array}$ \\
\hline Consejo Concertación & $\begin{array}{c}-0.210^{* * *} \\
(0.0306) \\
\end{array}$ \\
\hline Proyecto seguridad & $\begin{array}{l}0.453^{* * *} \\
(0.0536)\end{array}$ \\
\hline Proyecto cultura & $\begin{array}{c}0.347^{* * *} \\
(0.0449)\end{array}$ \\
\hline Fundación & $\begin{array}{c}0.179 \\
(0.126) \\
\end{array}$ \\
\hline Municipio & $\begin{array}{c}0.388^{* * *} \\
(0.0437)\end{array}$ \\
\hline Constante & $\begin{array}{c}16.98^{* * *} \\
(0.309)\end{array}$ \\
\hline Número de observaciones & 1.415 \\
\hline Número de grupos & 102 \\
\hline
\end{tabular}

Fuente: Elaboración propia con datos de los respectivos gobiernos regionales y SERVEL. ${ }^{* * *}$ significativo al $0.01,{ }^{* *}$ significativo al 0.05 .

Como muestra el cuadro $\mathrm{N}^{\circ} 4$, el tercer modelo de regresión selecciona a los partidos de la Concertación (PS-PPD-PRSD-PDC), y los partidos de la Alianza (UDI-RN), en lugar de la variable alcalde Alianza. La pobreza resulta ser significativa nuevamente, y la ruralidad aparece con signo negativo pero significativo, reproduciendo los resultados para ambas variables sobre la distribución de los fondos. Los resultados para los partidos políticos de la Alianza no tienen relación en la distribución de los montos. Las dos variables son negativas, pero ninguna es significativa, lo que indica que no reciben mayores fondos que los demás partidos. Para los partidos de la concertación en cambio, tres de los cuatro tienen algún grado de significancia en el proceso de asignación. Sin embargo, los tres partidos arrojan ser negativos. Lo que señala que poseen cierto grado de injerencia dentro de la distribución pero de 
manera que no reciben mayores montos; al contrario, reciben menos. Su rol como partido de oposición al gobierno de turno, determina esta significancia negativa que adquieren los partidos de la Concertación dentro del proceso de redistribución de los recursos (Cox \& McCubbins, 1986; Mediavilla, 2005).

\section{Cuadro 4. Tercer modelo regresión multinivel}

\begin{tabular}{|c|c|}
\hline \multicolumn{2}{|c|}{ Variable dependiente: Monto } \\
\hline Comunas bajo promedio pobreza nacional & $\begin{array}{l}0.242^{* * *} \\
(0.0684)\end{array}$ \\
\hline Comunas bajo promedio ruralidad & $\begin{array}{l}-0.213^{* *} \\
(0.0972) \\
\end{array}$ \\
\hline PDC & $\begin{array}{c}-0.282^{* * *} \\
(0.0963)\end{array}$ \\
\hline PPD & $\begin{array}{c}-0.00720 \\
(0.115)\end{array}$ \\
\hline PRSD & $\begin{array}{c}-0.258^{*} \\
(0.142) \\
\end{array}$ \\
\hline PS & $\begin{array}{l}-0.188^{*} \\
(0.112) \\
\end{array}$ \\
\hline $\mathrm{RN}$ & $\begin{array}{c}-0.0314 \\
(0.118) \\
\end{array}$ \\
\hline UDI & $\begin{array}{r}-0.0811 \\
(0.117) \\
\end{array}$ \\
\hline Consejo Concertación & $\begin{array}{c}-0.194^{* * *} \\
(0.0244) \\
\end{array}$ \\
\hline Proyecto seguridad & $\begin{array}{l}0.415^{\text {*** }} \\
(0.0354)\end{array}$ \\
\hline Proyecto cultura & $\begin{array}{l}0.327^{* * *} \\
(0.0286)\end{array}$ \\
\hline Fundación & $\begin{array}{l}0.277^{* * *} \\
(0.0885) \\
\end{array}$ \\
\hline Municipio & $\begin{array}{l}0.500^{* * *} \\
(0.0306)\end{array}$ \\
\hline Constante & $\begin{array}{c}16.81^{* * *} \\
(0.253)\end{array}$ \\
\hline Número de observaciones & 1.415 \\
\hline Número de grupos & 102 \\
\hline
\end{tabular}

Fuente: Elaboración propia con datos de los respectivos gobiernos regionales y SERVEL. *** significativo al $0,01,{ }^{* *}$ significativo al $0,05,{ }^{*}$ significativo al 0,1 . 
Para las demás variables del modelo (las mismas en las últimas dos regresiones) derivan los mismos resultados. El consejo de la Concertación continúa siendo negativo y significativo, lo que se traduce en las bajas sumas que reciben. Los proyectos de seguridad, con signo positivo y significativo, suman mayores montos en la distribución como ya hemos dicho. Con los proyectos de cultura sucede exactamente lo mismo. Este presenta ser significativo de manera que en la distribución de recursos le recaen considerables sumas. Por último, para las variables fundación y municipio los coeficientes coinciden con los resultados del modelo 1. Las dos son sobresalientes y de ello, que reciba el municipio, como la fundación, mayores niveles de fondos en el proceso de asignación.

Los resultados para los tres modelos coinciden en gran parte, y el comportamiento de las variables en el proceso de asignación demuestra ser el mismo. Salvo para algunas variables que tuvieron una variación de un modelo a otro, como el caso de la variable fundación; que para el modelo 2 presentó no tener ningún grado de significancia en la distribución del monto.

Para concluir el análisis inferencial vamos a examinar qué sucede con los tipos de proyectos cuando estos se transforman en variable dependiente. ¿Qué variables independientes son las que más se aproximan a cada proyecto? El cuadro $\mathrm{N}^{\circ} 5$ agrupa la serie de variables independientes que pretende explicar cuáles son las variables que tienen relación en la configuración de cada una de estas iniciativas.

La regresión logística para cada una de las variables dependientes, cultura, deporte, y seguridad, concluye que para el caso de los proyectos en cultura, solo tres de las ocho variables independientes, tiene significancia dentro del número de iniciativas. Comunas bajo el promedio de ruralidad, Partido Por la Democracia y Renovación Nacional son las variables que más se aproximan a proyectos de cultura. La variable más significativa es ruralidad, le sigue el Partido Por la Democracia y en último lugar, y de manera negativa, Renovación Nacional.

Para el caso de los proyectos deportivos sólo dos variables presentan significancia, pero negativa. Estas son pobreza y ruralidad. Ambas impactan negativamente en la configuración de iniciativas deportivas. Esto señala que en zonas rurales como en sectores más populares los proyectos en deportes no son llevados a cabo de manera significativa. Por último, en los proyectos de seguridad y pobreza, el Partido Radical Social Demócrata y Renovación Nacional resultan ser los únicos 
significativos, siendo el nivel de pobreza la variable de menor significancia, seguida por el PRSD, y RN, como la variable más significativa de las tres.

\section{Cuadro N5. Regresión Logística}

\begin{tabular}{l|l|l|l}
\hline Variables & Cultura & Deporte & Seguridad \\
\hline Comuna bajo promedio pobreza nacional & 0.114 & $-0.262^{* *}$ & $0.263^{*}$ \\
\hline Comuna bajo promedio ruralidad & $(0.121)$ & $(0.116)$ & $(0.150)$ \\
\hline & $0.476^{* * *}$ & $-0.451^{* * *}$ & 0.0594 \\
\hline Partido Demócrata Cristiano & $(0.149)$ & $(0.137)$ & $(0.171)$ \\
\hline & -0.0710 & -0.0421 & 0.183 \\
\hline Partido Por la Democracia & $(0.160)$ & $(0.152)$ & $(0.197)$ \\
\hline & $0.487^{* *}$ & -0.300 & -0.401 \\
\hline Partido Radical Social Demócrata & $(0.224)$ & $(0.226)$ & $(0.337)$ \\
\hline & -0.0950 & -0.322 & $0.606^{* *}$ \\
\hline Partido Socialista & $(0.279)$ & $(0.264)$ & $(0.303)$ \\
\hline Renovación Nacional & -0.0697 & 0.117 & -0.104 \\
\hline & $(0.175)$ & $(0.168)$ & $(0.230)$ \\
\hline Unión Demócrata Independiente & $-0.643^{* * *}$ & -0.328 & $1.088^{* * *}$ \\
\hline & $(0.234)$ & $(0.209)$ & $(0.227)$ \\
\hline Constante & 0.0103 & -0.119 & 0.175 \\
\hline & $(0.214)$ & $(0.204)$ & $(0.260)$ \\
\hline Número de observaciones & $-1.009^{* * *}$ & $0.383^{* *}$ & $-1.803^{* * *}$ \\
\hline & $(0.164)$ & $(0.152)$ & $(0.200)$ \\
\hline
\end{tabular}

Fuente: Elaboración propia con datos de los respectivos gobiernos regionales y SERVEL. ${ }^{* * *}$ significativo al $0.01,{ }^{* *}$ significativo al 0.05 .

\section{Conclusión}

En esta investigación se ha intentado comprobar de qué manera y hasta qué punto la distribución de recursos es mediada por factores políticos. Dentro de la exposición de las teorías y visiones sobre la redistribución política hemos contrastado y validado las diversas interrogantes respecto a este tipo de redistribución. A lo largo del estudio hemos presentado y caracterizado la dinámica política dentro de la distribución del 2\% del Fondo Nacional de Desarrollo Regional en cuatro regiones del país, tomando como referencia las visiones de los diferentes autores 
en el tema. Sobre esto, afirmamos una tendencia hacia el manejo político de los recursos que se identifica con algunas de las nociones planteadas en la teoría, vale decir, que la cercanía al gobierno de turno favorece la distribución de beneficios (Cox \& McCubbins, Grossman, Mediavilla, entre otros). Como también la captación hacia votantes indecisos (Dixit \& Londregan y Lindbeck $\&$ Weibull), que tiende a buscar réditos políticos en los sectores más vulnerables de la población. En este sentido, a mayores índices de pobreza más incidencia en el reparto de los recursos de acuerdo a la distribución llevada a cabo por los Gobiernos Regionales.

Un hallazgo interesante es el vínculo según el tipo de iniciativas y la posición política del alcalde, en comunas con alcaldes de la Alianza los proyectos de seguridad pública lideran el porcentaje de iniciativas. Caso contrario ocurre en las comunas presididas por un alcalde de la Concertación; en estas los proyectos en cultura se adjudican más de un tercio de los tres tipos de iniciativas. Cabe preguntarnos entonces, ¿juega la ideología una implicancia en aquello?

Otro punto importante, es lo que sucede en zonas rurales. Aquí se presentan altos índices de pobreza, y sin embargo, esto no se condice en el reparto que estas zonas se adjudican, ya que los montos destinados resultan ser escasos.

Al llevar a cabo el análisis de los Gobiernos Regionales nos permitimos determinar tres tipos de discrecionalidad. La primera, parte de las falencias regionales como necesidades no resueltas por parte de los Gobierno Regionales. Lo que lleva a que exista un reparto de los fondos de inversión de manera discrecional, direccionando hacia las comunas con mayores niveles de pobreza en contraste con las comunas en mejor situación. Esta discrecionalidad socioeconómica se transforma en la más determinante a la hora de distribuir los recursos desde el gobierno regional a los gobiernos locales. Ello fue confirmado para cada una de las regiones de estudio. Segundo, una discrecionalidad de carácter organizacional inyecta mayores montos para los municipios en comparación con el resto de organizaciones, salvo las fundaciones y clubes, que reciben flujos importantes. En tercer lugar, y la más relevante al caso, la discrecionalidad política, valida la existencia de cierta distribución discrecional para las comunas presididas por alcaldes de la Concertación, como también, para las regiones con mayor número de consejeros regionales de la Concertación. Estas demostraron recibir 
menores sumas de fondos en comparación con los alcaldes de la Alianza, aun cuando las comunas presididas por estos no recibieran mayores niveles de monto en comparación conjunta con los demás alcaldes. Este hecho resulta relevante, porque el que comunas con alcaldes de la Alianza reciban mayor promedio de monto que comunas presididas por la Concertación (que las superan en número), deja entreabierta la existencia de que al ser menor el número de comunas de la Alianza, se pretenda generar algún tipo de protección hacia estas.

A modo de cierre, es preciso señalar que la distribución desarrollada por los Gobiernos Regionales no sólo se aboca a responder a carencias territoriales, sino que también se transforma en un instrumento de uso táctico para la redistribución de beneficios, ya sean estos sociales o económicos, el fin termina siendo el mismo: la búsqueda de una ganancia política. En suma, debemos agregar que si bien nuestra hipótesis no satisface por completo la aseveración de que las distribuciones llevadas a cabo por los gobiernos regionales responden a criterios netamente políticos, por sobre criterios socioeconómicos y territoriales, no podemos desecharla de lleno. Por lo tanto, se deja un espacio a la incertidumbre al pensar qué sucedería en un contexto de análisis nacional; ¿persistiría la discrecionalidad socioeconómica de igual manera para todas las regiones del país? ¿Tomaría relevancia la discrecionalidad política?, sin duda esto abre un espacio para llevar a cabo nuevas investigaciones que den a conocer la realidad en los diferentes territorios de nuestro país.

\section{BiBLIOGRAFÍA}

Brusco, V. y Nazareno, y Stokes (2005). "La manipulación política de los recursos públicos: réditos y costos electorales en Argentina”. Ponencia presentada en VII Congreso Nacional de Ciencia Politica, Sociedad Argentina de Ciencia Política.

Cox, G. y M. McCubbins (1986). "Electoral Politics as a Redistributive Game". The Journal of Politics, Vol. 48, No2, pp. 370-389.

Dahlberg, Matz y Eva Johansson (2002). "On the Vote Purchasing Behavior of Incumbent Governments”. The American Political Science Review Vol. 96, No1, pp. 27-40.

Dixit, A. y John L. (1996). "The Determinants of Success of Special Interest in Redistributive Politics". The Journal of Politics, Vol. 58, No4, pp. 1132-1155. 
Grossman, Philip (1994). "A Political Theory of Intergovernmental Grants". Public Choice 78, No3/4, pp. 295-303.

Inman, Robert (1988). "Federal Assistance and Local Services in the United States: The Evolution of a New Federalist Fiscal Order". NBER Working Paper No 2.283.

Johansson, Eva (1999). "Intergovernmental Grants as a Tactical Instrument; Some Empirical Evidence from Swedish Municipalities". Uppsala-Working Paper Series No 10.

Llancar, Carlos (2009). "Descentralización: la experiencia chilena, un estudio sobre los gobiernos regionales". Revista de Estudios Politécnicos Vol. VII, No11.

Mediavilla, Mauro (2005). "Determinantes políticos de las transferencias discrecionales; evidencia de la provincia de Córdoba, Argentina”. Revista de Economía y Estadistica, Cuarta Época, Vol. 43, No1, pp. 167-192.

Oates, Wallace (1973). Fiscal Federalism. New York: Harcourt Brace Jovanovich.

Schady, Norbert (2000). "The Political Economy of Expenditures by the Peruvian Social Fund (FONCODES), 1991-1995”. American Political Science Review Vol. 9, No2, pp. 289-304.

SUBDERE (s/f). El financiamiento de la inversión pública regional y municipal: el fondo nacional de desarrollo regional y el fondo común municipal. Gobierno de Chile.

Weitz-Shapiro, Rebecca (2006). "Partisanship and Protest: The Politics of Workfare Distribution in Argentina”. Latin America Research Review Vol. 41, No 3, pp. 122-147.

Wright, G. (1974). “The Political Economy of New Deal Spending: An Econometric Analysis”. The Review of Economics and Statistics, Vol. 56, No1, pp. 30-38. 